\title{
Deviation of a machined surface in flank milling
}

\author{
A. Larue ${ }^{\mathrm{a}, *}, \mathrm{~B}$. Anselmetti ${ }^{\mathrm{a}, \mathrm{b}}$ \\ a Laboratoire Universitaire de Recherche en Production Automatisée, Ecole Normale Supérieure de Cachan, 61, av du président Wilson, 94235 \\ Cachan Cedex, France \\ ${ }^{\mathrm{b}}$ Institut Universitaire de Technologie de Cachan, 9, av de la division Leclerc 94234 Cachan Cedex, France
}

\begin{abstract}
The flatness defects observed in flank milling with cutters of long series are mainly due to the tool deflections during the machining process. This article present the results of an identification procedure of the coefficients of a force model for a given tool workpiece couple for the prediction of the defects of the tool during the cutting. The calibration method proposed meets a double aim: to define an experimental protocol that takes the industrial constraints of time and cost into account and to work out a protocol which minimizes uncertainties likely to alter the interpretation of the results (environmental, software or mechanical uncertainties). For that, the procedure envisages the machining of a simple plane starting from a raw part formed by a tilted plane, allowing for the variation of the tool engagement conditions. The tool deviation during the cutting process is indirectly identified by measuring the machined surface. The observed straightness defect conditions can be explained by the evolution of the cutting pressures applied to the cutting edges in catch during the cutter rotation. The precision was considerably improved by the taking into account of the cutter slope defect in the calculation of the load applied to the tool. After identification of the tool-workpiece couple, the prediction model was applied to some examples and allowed to determine the variations of form and position of the surface points with a margin of $5 \%$.
\end{abstract}

Keywords: Cutter deformation; Force model; Flank milling; CAM integration

\section{The milling}

\subsection{Tool paths generation in flank milling}

'Machining tool paths generation' is to be carried out by seeking the best possible geometrical quality on the machined part while reducing times of manufacture. The context of our study is that of the flank milling of free forms parts with long tools. This very efficient process from the point of view of productivity and of surface quality is very much used in aeronautics and mould manufacturing.

Flank milling of free surfaces traditionally implies the generation of machining tool paths that will ensure an optimal laying of the presumed rigid tool on the surface

\footnotetext{
* Corresponding author. Tel.: +33-1-47-40-27-65; fax: +33-1-4740-22-20.

E-mail address: larue@lurpa.ens-cachan.fr (A. Larue).
}

to machine [1-4]. In flank milling with long tools, the geometrical errors generated by the tool deflection during the cutting process can be very significant. Thus, to obtain a surface of quality, it is necessary to take the tool deformation under the cutting forces into account.

To foretell the tool deflections, this article presents a method of identification for a tool workpiece couple in flank milling. The experimental protocol is easily applicable industrially. The data-processing exploitation of the prediction model allows its integration in a $\mathrm{CAD} / \mathrm{CAM}$ environment.

Before detailing the identification procedure, let us briefly come back on to the choice of force model.

\subsection{Choice of force model}

The numerous existing force models can be classified into two modeling levels: 'microscopic modelisation' studies the interaction between the tool and the workpiece material by using thermomechanical behavior laws 
and 'global modelisation' considers the force resulting from the contact between the tool and the part along the cutting edges in catch.

The microscopic analysis of the chip formation makes it possible to understand the physical phenomenon of the material removal to apprehend the thermomechanical phenomena generated during the cutting of the workpiece material. The numerical simulation of machining has to be very precise and requires a good knowledge of the tool (sharpness of the cutting edge, forms of the groove, ...). This type of approach is for the moment essentially used in turning $[5,6]$ and can lead to the improvement of tool quality. The time simulation of these approaches remains relatively significant, sometimes exceeding the hour of calculation. The integration of a microscopic model in a CAD/CAM environment of tool paths generation thus seems easier by using a 'macroscopic' force model.

The 'macroscopic' models describe the cutting phenomenon by studying the evolution of the force resulting from the chip formation along the cutting edges in catch. These models are divided into two categories according to whether they take the time in the machining simulation into account or not. The approaches known as 'dynamic' consider the instantaneous depth of cut variation between the trajectory of the tooth which machines and the preceding tooth. These models are based on the regeneration mechanism of the machined surface, considered as the main cause of the vibrations appearance [7-10].

The objective of the 'dynamic' models is to envisage the appearance of instabilities which cause chatter during the machining process. The dynamic behavior of the tool and/or part are thus described either by linearizing the cutting pressures laws to study instabilities in the frequential field [11-14], or by describing the geometry of the machined surface step by step in the temporal field $[9,10,15]$.

The static approaches are based only on the concept of cutting pressure applied to a theoretical chip section and on the fixed beams theory to estimate the deformations. In milling, the Kline and DeVor's model [16] has already been used by Seo [17] to treat flank milling and the problems of machining tool paths compensation.

The context of the study proposed in this article is that of flank milling at traditional cutting speed lower than $200 \mathrm{~m} / \mathrm{min}$. The rotational frequency of the spindle is low. We thus chose to adapt the Kline and DeVor's model for great axial engagements.

The article is organized as follows: section 2 describes the model which is at the basis of the identification procedure. Section 3 details the experimental procedure and the results obtained. Section 4 finally reconsiders specificities of the calculation of the angle of the tool real engagement into the workpiece material, which is a significant contribution of the method proposed, by show- ing that the fact of taking the tool deflection into account in this calculation improves the precision of the identification.

\section{Modelisation of the deformation}

This article is only interested in flank milling of steels at traditional speeds with solid plain milling cutters whose cutting edges are helicoid. The defects of the surfaces machined with long series cutters are very significant: localization defect of $0.8 \mathrm{~mm}$, flatness defect of 0.2 $\mathrm{mm}$. Taking the test conditions with a rigid part, a rigid assembly and a rigid machine into account, we suppose that the main cause of deformation is due to the tool deflection.

\subsection{Qualitative analysis of the cutting process}

Modeling the cutting process requires the understanding of the associated physical phenomenon. During a flank milling process, the locations of the cutting edges in catch simultaneously evolve in the workpiece material. The machined surface is thus obtained by a generating point $P$ which moves along the cutting generator while the tool rotates.

For a given angle, the generating point is in $P$ at a distance $z$ of the spindle nose considered as a housing. After a small rotation of the plain milling cutter, the generating point is in $P^{\prime}$ at a distance $z^{\prime}$ (Fig. 1). This explains the variation of the radial forces and of the tool deformations.

An elementary cutting edge placed at point $Q$, which is in the workpiece material, cuts an elementary chip section by applying an elementary force $d F$. At each point $\mathrm{P}$, the resulting deformation is the sum of the elementary deformations caused by the engagement of all the elementary lengths constituting the cutting edges simultaneously engaged in the workpiece material. Fig.

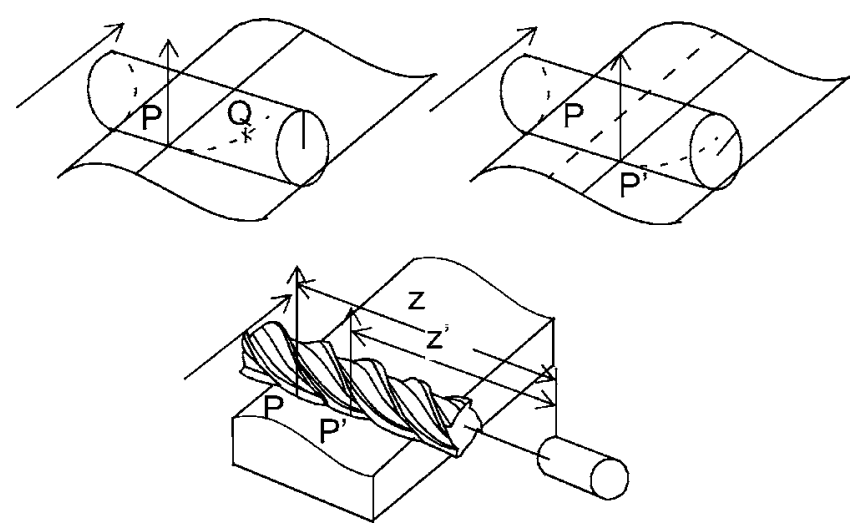

Fig. 1. Evolution of the generating point of a tool. 

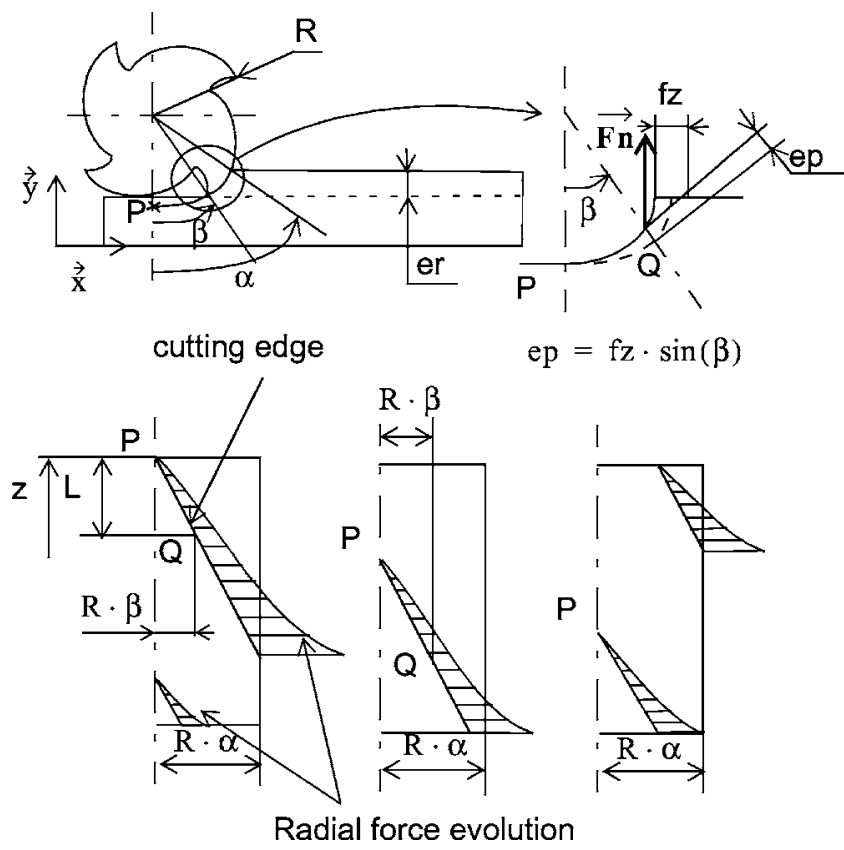

Fig. 2. Force along a cutting edge according to the cutter angular position.

2 represents the evolution of radial force per unit of length along a helicoid developed profile on the machined surface. $\alpha$ is the total tool engagement angle in the workpiece material. Each point $Q$ is characterized by the angular position $\beta$ and by its distance $z$ to the spindle nose.

The variation of the radial forces and that of the distances from point $P$ to the presumed housing along a machined section makes it possible to qualitatively understand that the resulting deformation of the section is not linear.

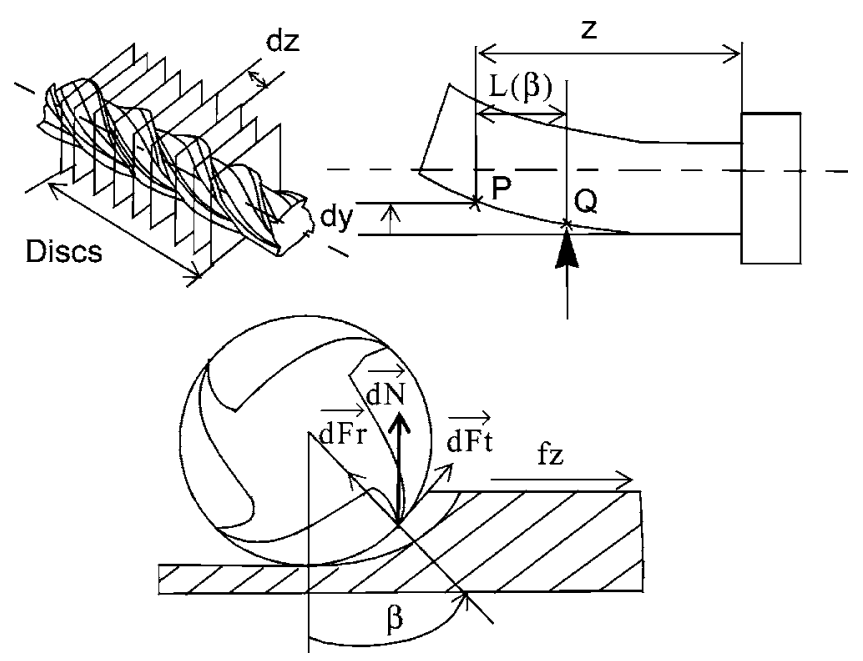

Fig. 3. Tool deformation.

\subsection{Definition of the deformation model}

The calculation of the cutting pressures is approached by making use again of the Kline and DeVor's model [16]. We hence propose a force model based on the decomposition of the tool into elementary discs the thickness $d z$ of which is considered as constant. Each elementary force applied in each point $Q$ of the cutting edge in catch considered is decomposed into a tangential component $d F t$ and a radial component $d F r$ :

$d F r=K r . e p \cdot d z=K r_{o} \cdot(f z \cdot \sin (\beta)) \cdot d z \cdot(f z \cdot \sin (\beta))^{-0.3}$
$d F t=K t . e p \cdot d z=K t_{o} \cdot(f z \cdot \sin (\beta)) \cdot d z \cdot(f z \cdot \sin (\beta))^{-0.3}$

Specific cutting pressures $K r$ and $K t$ are supposed to answer the following model:

$K r=K r_{0} \cdot e p^{-0.3}$ and $K t=K t_{0} \cdot e p^{-0.3}$

where $e p$ is the depth of cut given by $e p=f z \cdot \sin (\beta)$.

This model was many times validated in the context of turning, coefficient -0.3 appearing as a rather stable constant.

The geometrical variations of the machined surface are mainly due to the plain milling cutter deformation in the normal plane to the surface. Only the component $d N$ of this cutting force interests us.

$d N(\beta)=d F r(\beta) \cdot \cos (\beta)+d F t(\beta) \cdot \sin (\beta)$.

We consider that the tool is a fixed beam of constant moment of inertia $I_{g z}$, that is the case when the tool used has a teeth number multiple of 4 . The resulting deformation $d y$ at point $P$ from coordinate $z$, due to the radial force $d N$ applied to point $Q$ is given by:

$d y=\frac{1}{E \cdot I_{g z}} \cdot d N(\beta) \cdot\left[\left(\frac{(z-L(\beta))^{3}}{3}\right) \cdot\left(\frac{(z-L(\beta))^{2} \cdot L(\beta)}{2}\right)\right]$

where $L(\beta)=(h \times \beta) /(2 \pi)$ if $Q$ belongs to the same tooth as $P, h$ being the helicoïdal pitch and $E$ being the Young's modulus of the tool. The moment of inertia is given by $I_{g z}=\pi \cdot d_{e q}^{4} / 64$ with $d_{e q}=\mu \cdot 2 R, R$ being the plain milling cutter radius and $\mu$ an equivalence ratio identified during a static test similar to the test presented in Fig. 5. An error in the determination of $I_{g z}$ has no influence on the identification precision as this error is made up for at the time of the matrix system resolution leading to the specific cutting coefficients values $K r_{0}$ and $K t_{0}$.

The total theoretical deformation $y t h_{i}$ at point $P_{i}$ is the sum of the elementary deformations dy generated by all the points $Q$ of the cutting edges engaged simultaneously in the workpiece material.

$y t h_{i}=\sum_{k=1}^{N} \int_{(\beta=0)}^{(\beta=\alpha)} d y_{\mathrm{ik}}=\sum_{k=1}^{N}\left(\frac{1}{E \cdot I_{g z}} \int_{(\beta=0)}^{(\beta=\alpha)} d m f_{i k}\right)$

where the bending moment $d m f_{\mathrm{ik}}$ is given by: 


$$
\begin{gathered}
d m f_{i k}=d N(\beta) \cdot\left(\left(\frac{\left(z_{i}-L(\beta)_{i k}\right)^{3}}{3}\right)\right. \\
\left.+\left(\frac{\left(z_{i}-L(\beta)_{i k}\right)^{2} \cdot L(\beta)_{i k}}{2}\right)\right)
\end{gathered}
$$

where $i$ is the index of point $P_{i}$ along the studied line, $k$ is the number of the considered cutting edges and $N_{t}$ is the number of the teeth of the plain milling cutter.

The engagement angle $\alpha$, useful for the integral calculation Eq. (5) depends on the cutting edge and on the tool orientation. During this study, a basic model was used by considering that the angle $\alpha$ is constant and calculable for a radial engagement er given by $\alpha=$ $a \cos ((R-e r) / R)$. We then noted rather significant differences between measurements and the estimates of variations of the surface machined. We observed that under effect of the the tool deformations, the maximum engagement angle $\alpha$ considerably decreases. It was thus necessary to set up a process to take the deformation in the engagement angles calculation into account. Section 4 will specifically detail this point.

Ultimately, the approach proposed in this article extends the Kline and DeVor's model [16] to the taking into account of great axial engagements. The integral relates to the deformation due to each elementary cutting edge length whereas, in the original model, the integral relates to the forces, the resultant forces being used to calculate the deformation.

\section{Identification procedure}

\subsection{Introduction}

The determination of the force model coefficients is a significant stage for the prediction of the machining defects. The estimate of these parameters classically requires the realization of a significant campaign of tests during which the cutting pressures are generally measured with dynamometers [18-24]. These experimental data are then treated by an optimization method and lead to the determination of the coefficients. The results obtained always depend on the conditions of realization of the tests and on the hypotheses of calculation set up.

The most current identification strategies concern the direct measurement of characteristic components of the cutting process for a tool-workpiece couple $\left(K r_{0}, K t_{0}\right.$ characterized) for given conditions. The measurement of the force is difficult in an industrial cycle, because it requires the maintainance of a cutting force turntable, its central processing unit and a very qualified technician. The examination is also rather delicate because there are generally several teeth in catch. After that, it is necessary to associate a tool deflection model to the phenomenon resulting from the presumed cutting forces.
To define a protocol of identification answering the industrial constraints, we use an indirect measurement by quantifying the tool deflection with the measurement of the machined surface. The opposite problem is then solved by comparing the theoretical deformation of the tool with the deformation of the machined surface.

In this context, Landon and al. propose the machining and the measurement of a master part gathering characteristic machinings from which we can infer a law governing the machining defects [25]. The approach proposed can be applied to all the types of machining and is not based on a theoritical force model.

\subsection{Principles of the procedure}

To make the identification procedure easily reproducible industrially while reducing uncertainties related to its implementation and its interpretation, a part should be defined that allows to characterize a tool workpiece couple on a given machine in stabilized mode.

\subsubsection{Principles of the test selected}

Taking the constraints previously evoked into account, we define a test protocol based on the flank milling in concordance of a simple plane which is realizable on any milling machine. To vary the depth of cut of 0.5 up to $3 \mathrm{~mm}$, the raw part has a specific form mainly constituted of a tilted plane (Fig. 4) and of surfaces dedicated to the realization of references to facilitate measurements.

The raw part is divided into four zones:

- In the identification zone, the raw surface is a tilted plane giving a depth of cut variation which is included between 0.5 and $3 \mathrm{~mm}$

- The reference zones which require two narrow bands

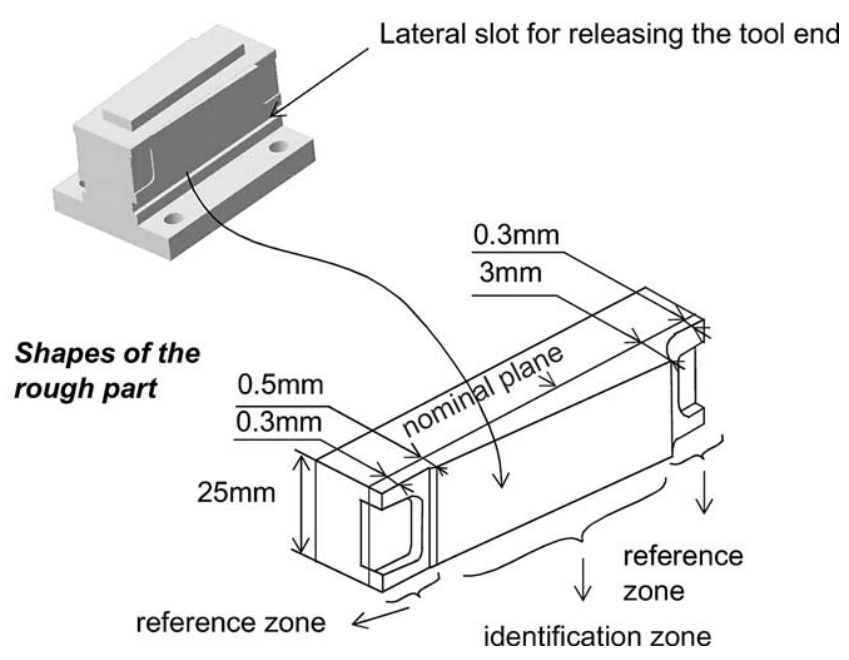

Fig. 4. Specifications of the raw part. 
with a small depth of cut of $0.3 \mathrm{~mm}$ in order to limit the cutting forces and the tool deflections

- A lateral slot that lets the tool end out of the workpiece material.

In order to isolate the influence of the tool deflection on the part defect, the tool end is left free to avoid the disturbances caused by a parasitic friction of the tool end.

\subsubsection{Experimental validation of the assumptions}

To obtain an experimental model representing the cutting process as precisely as possible, the following hypotheses are posed:

1. The part is very rigid and firmly taken in on the part holder.

2. The part holder is very rigid.

3. The tool is considered as a fixed beam.

4. The spindle of the machine unit is considered as a rigid body.

To quantify the spindle deformation, a force of the same order of magnitude (maximum $150 \mathrm{~N}$ ) as the current cutting force is applied on a very rigid test holder, using a dynamometric ring. The induced deflection is obtained using two comparators for one point of load (Fig. 5). The deviation of the spindle axis on the level of the tool end is about $6 \mu \mathrm{m}$, which is negligible compared to the observed variations on the part. In static, the spindle can thus be regarded as a rigid bogy. The point $\mathrm{A}$, located near the spindle nose, is retained as the presumed housing point.

To analyze the rigidity of the machine, a comparator is fixed on the spindle casing during the preceding

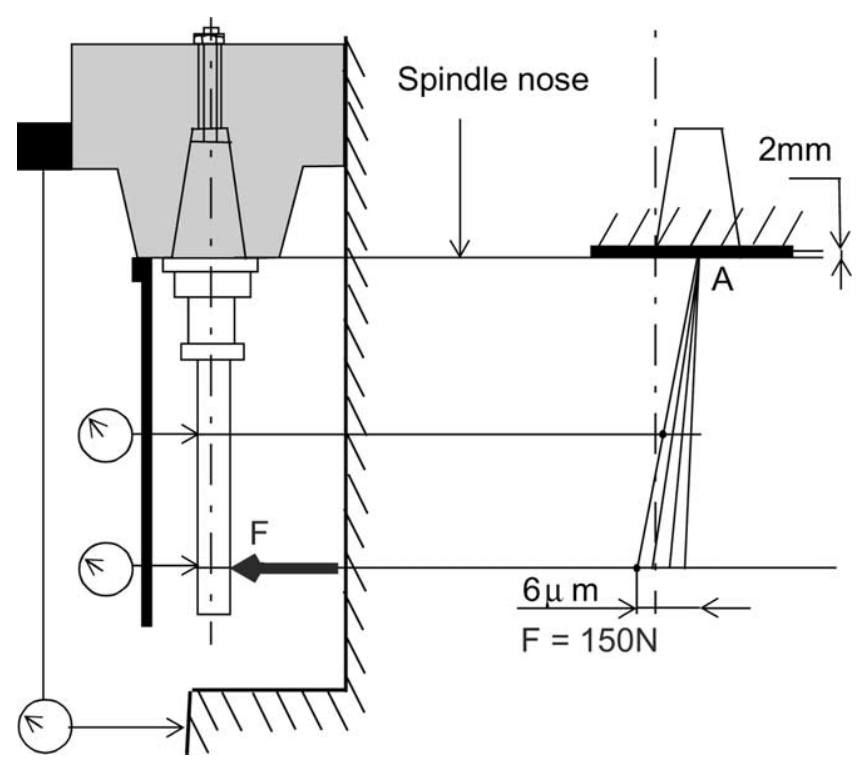

Fig. 5. Validation of the static rigidity of the machine. identification test to measure the displacements of the machine table compared to the spindle. The maximum deformation noted is lower than $15 \mu \mathrm{m}$ and will be neglected too.

We finally consider that the dynamics of the machine spindles does not disturb machining, because the machined surface is a simple plane which is milled at constant feedrate with a long stabilization and acceleration distance before machining the central part (section 3).

\subsubsection{Principles of measurement}

From an operational point of view, the identification consists in machining the test part and measuring the defects of the machined surface. These measurements make it possible to establish a surface statement of the deformations. This statement gives the actual value of the deformation at each point of a measurement grid posed on the machined surface. The step of the grid is of $1 \mathrm{~mm}$.

To measure the defects of the test part correctly, a reference mark was conceived on the basis of reference surfaces (Fig. 6). This reference mark is built by bypassing the part with the tested tool by taking a weak depth of cut of $0.3 \mathrm{~mm}$ on an axial engagement of 5 $\mathrm{mm}$. It thus allows for an effective registration between the reference mark part and the reference mark of the CMM machine. This type of machining allows the construction of reference surfaces while being freed from the gauge and tool deflection defects. The two zones with low depth of cut located at the level of the part ends are used to validate the registration of the measured points cloud.

Within the framework of this article, the part test was machined under the following conditions:

- HSS tool whose diameter is $20 \mathrm{~mm}$ and whose active length is $88 \mathrm{~mm}$

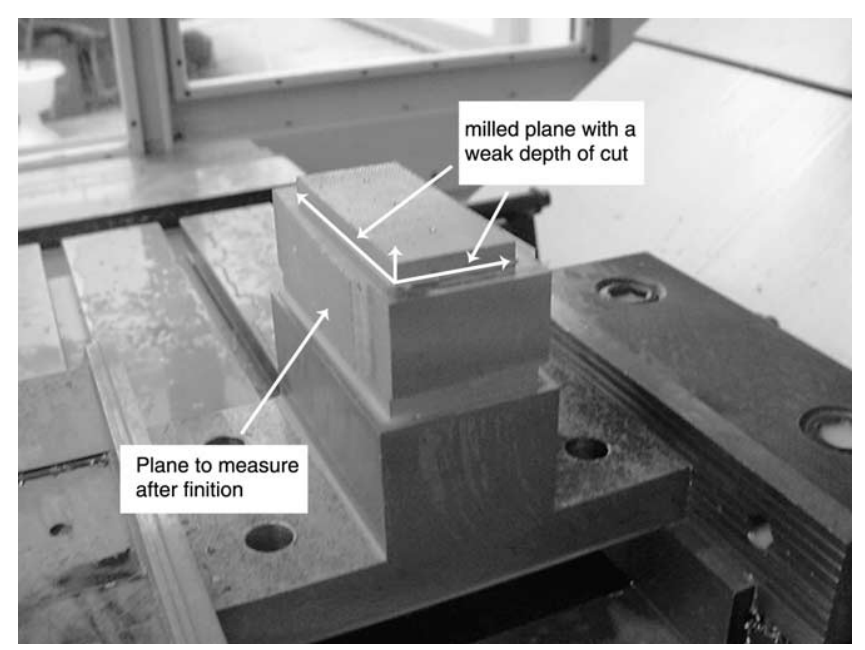

Fig. 6. Reference mark useful for measurement. 
- Machining on a vertical milling center

- $\mathrm{Vc}=25 \mathrm{~m} / \mathrm{min}$

- $\mathrm{fz}=0.2 \mathrm{~mm} /$ tooth

- $\mathrm{Nt}=4$ teeth.

\subsection{Experimental results}

Machining revealed the presence of a flatness defect whose shape is a wave, reflecting the varying distribution of the forces in time. Only the central zone of the cloud of measured points corresponding to the permanent mode (represented on the Fig. 7) is used to identify the deflection model.

The machined plane is tilted and curved with a flatness of $0.2 \mathrm{~mm}$. The observed deviation increases with the depth of cut.

For a depth of cut of $3 \mathrm{~mm}$, the maximal deviation is $0.7 \mathrm{~mm}$.

In order to determine the pressure coefficients, we use a method of least squares which minimizes the sum of the squared differences between the deformation measured and the one calculated theoretically thanks to the model previously described, for each point of the grid which is posed on the surface to machine. The coefficients $K r_{O}$ and $K t_{0}$ are obtained by canceling the partial derivative, which leads to the resolution of a matrix system:

$\frac{\delta W}{\delta K r_{0}}=\frac{\delta W}{\delta K t_{0}}=0$

where
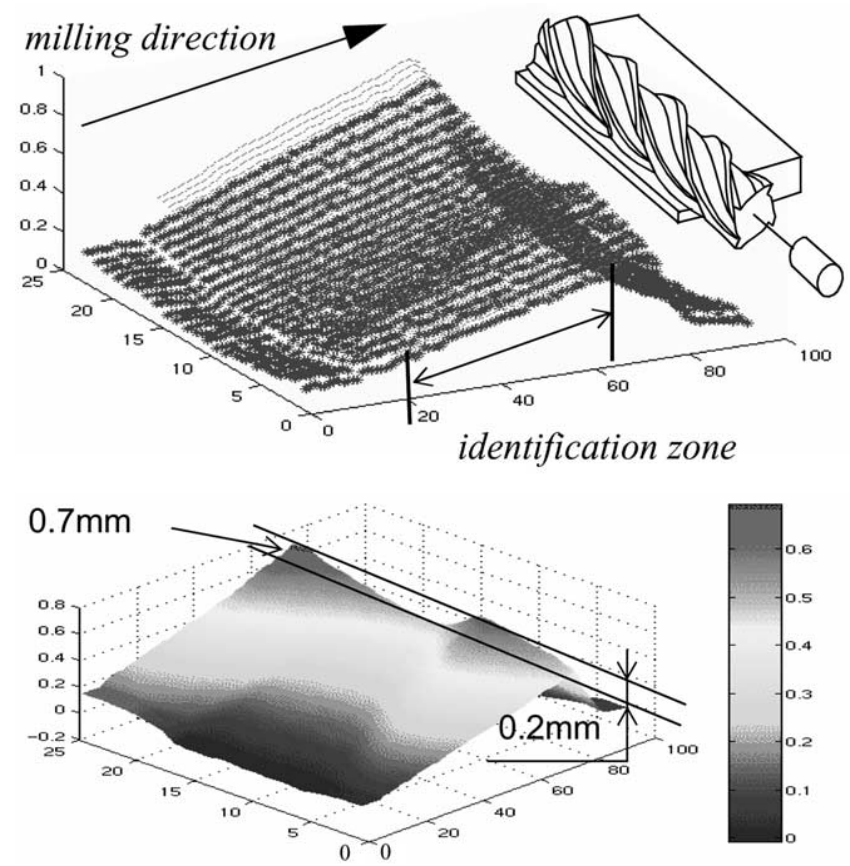

Fig. 7. Selection of the points being used in the identification stage.
$W=\sum_{m=1}^{N s} \sum_{i=1}^{N d}\left(y t h_{i m}-y m e s_{i m}\right)^{2}$

where $N s$ is the number of sections taken into account on the surface measured and $N d$ is the number of points taken on each section.

$y^{y m e s}{ }_{i m}$ is the measured deflection at the grid point of index $i m$ and $y t h_{i m}$ is the theoretical deflection in this point, calculated thanks to the preceding Eq. (8).

A software model makes it possible to solve the preceding system. The coefficients $K t_{o}$ and $K r_{o}$ of the force model are thus obtained and make it possible to predict the machining defects whatever the case of machining. $K r_{0}=318 ; K t_{0}=103$.

Fig. 8 illustrates the error between the profile measured and the simulated one. The error is included between $+0.05 \mathrm{~mm}$ and $-0.05 \mathrm{~mm}$. The average of uncertainty on each predicted point is of $-0.001 \mathrm{~mm}$. The static model used thus makes it possible to predict the deformation obtained in flank milling with an error of $5 \%$ at classical speed.

The preceding test was carried out for cutting speeds ranging from 18 to $25 \mathrm{~m} / \mathrm{min}$ by preserving the same feedrate and thus appreciably the same cutting pressures. In spite of a light effect due to the variation of the tool workpiece couple according to the cutting speed, each test made it possible to note that the variations of the points and the form of the defects previously noted were quasi imperceptible to speed thus to time. We conclude that the machining defects observed are certainly not caused by a phenomenon which originates in the vibrations. Nevertheless, the error profile (Fig. 8) is marked by a low undulatory defect according to the radial depth of cut. This phenomenon is perhaps related to a regenerative effect along the surface, phenomenon which is not taken into account in our model. If one takes the maximum measurement uncertainty into account, estimated at $0.05 \mathrm{~mm}$ to the measure of a plane, the results obtained are acceptable.

The test which has just been described in this section

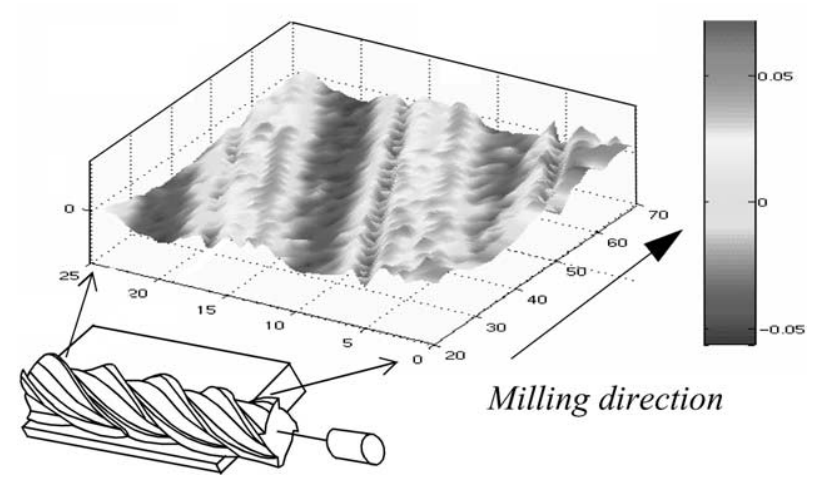

Fig. 8. Comparison of simulated and machined surfaces. 
was also carried out with a shorter carbide tool [26]. The results obtained are similar.

\section{Calculation of the conditions of engagement}

\subsection{Originality of the method}

In section 2, we observed that at the time of the tool deformation, the engagement angle $\alpha$ considerably varies. It is thus necessary to know the deformation to estimate the engagement angle in order to better identify the deformation. The originality of this work lies in the consideration of this paradox and on its resolution by avoiding iterative procedures.

Fig. 9 goes back onto the various stages of the calculation of the theoretical tool deformation. This calculation begins with the installation of a grid of points on the surface to machine. The normal differences between the nominal plane and the machined plane are then measured for each point of this grid. After interpolation of cloud of measured points, we obtain the surface distribution of the radial variations. To calculate the tool deflection at each point of the grid, it is necessary to first determine the tool engagement angles in the workpiece material. We then simultaneously determine the position of the teeth in catch when the point generated is one of the points of the grid by using an evolution model of the teeth location similar to Choi's [27]. For each point of the grid, the configuration of the teeth in catch is known and makes it possible to determine the input angles ('min angle') and the output angle of the workpiece material ('max angle') by using intersection calculations between the tool helix and the workpiece material. Once the engagement angle is known, the tool deflection can be calculated for each point of the grid by using the model previously explained (paragraph 2.2). The surface distribution of the variations on the part rep-

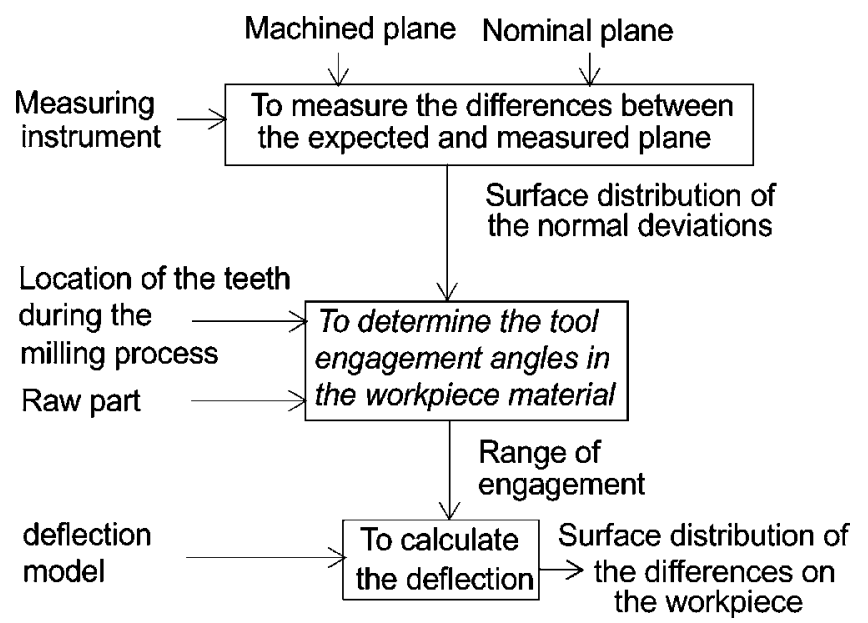

Fig. 9. Deformation computing process for the identification of the tool workpiece couple.

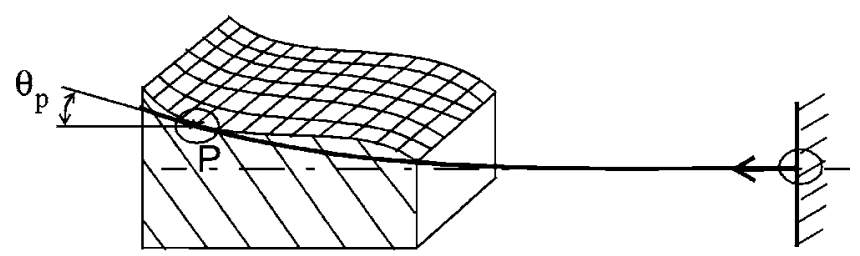

\section{Deformation model of the tool considered as a rigid body}

Fig. 10. Curve passing by the tool characteristic points.

resenting the tool deflection along the machined surface is then obtained.

\subsection{Calculation of the engagement angles}

This paragraph aims to specify the details of the calculation of the engagement angles according to the tool deformation. The form of the tool, considered as a fixed beam, is modelized for each machined section by a 2 or 3 degree polynomial curve which is tangent to the housing (Figs. 10 and 11) and which contains one or two points of the profile of the deflection according to if in the section considered there are one or two teeth engaged at the same time. Fig. 10 presents a case where there is one tooth engaged in the workpiece material.

For each point of the grid, the tool slope is given by considering the slope $\theta_{p}$ of the interpolation curve representing the tool deformation. The tool generator point $P$ is placed on $y_{i m}$.

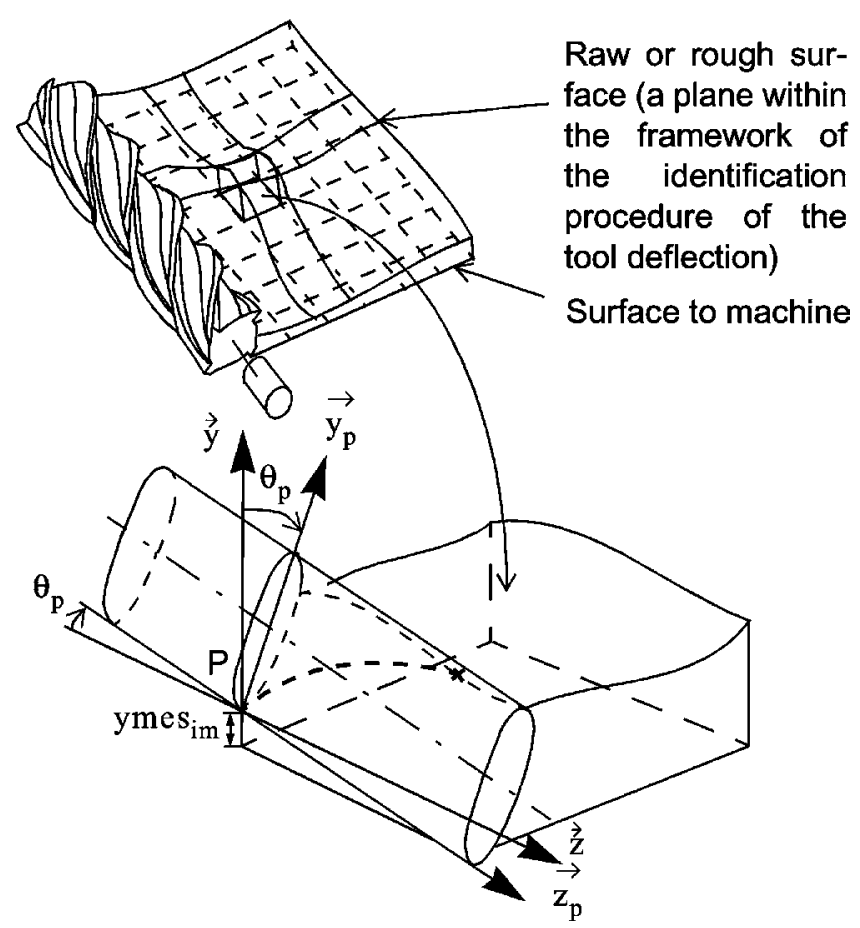

Fig. 11. Tool modelization on a machined section. 
During machining, the tool leans under the cutting forces. The tool slope must be taken into account in the calculation of the extreme point position. For that, a rotation is applied to the previously defined curve in order to know the real position, which take the deformation undergone by the tool into account (Fig. 12).

To determine the location of the exit point of the cutting edge, we use a parametric representation of the cutting edge. To each machined position of the finished sur-

face is associated a local reference mark $\left(\mathrm{P}, \vec{x}_{p}, \vec{y}_{p}, \vec{z}_{p}\right)$ where $\vec{x}_{p}$ is the unit vector indicating the machining direction, $\vec{z}_{p}$ is the unit vector representing the tool axis and $\vec{y}_{p}$ is the vector defined such as $\vec{y}_{p}=\vec{z}_{p} \wedge \vec{x}_{p}$. In this reference datum, the cutting edge is defined without taking the deformation into account by:

$x=r \times(\sin (2 \pi \times t / h))$

$y=r \times(1-\cos (2 \pi \times t / h))$

$z=t$.

In the reference mark $\left(P, \vec{x}_{p}, \vec{y}_{p}, \vec{z}_{p}\right)$.

$x=r \times(\sin (2 \pi \times t / h))$

$y=r \times \cos \left(\theta_{p}\right) \times(1-\cos (2 \pi \times t / h))+t \times \sin \left(\theta_{p}\right)$

$z=-r \times \sin \left(\theta_{p}\right) \times(1-\cos (2 \pi \times t / h))+t \times \cos \left(\theta_{p}\right)$.

Fig. 12 shows that the slope of the tool modifies the length of the cutting edge in catch, which yields a noticeable modification of the engagement angle $\alpha$.

The coordinates of the exit point of the tool cutting edge are calculated by intersection between the helix and the rough surface. We thus obtain $\alpha=2 \pi . t_{\max i} / h$.

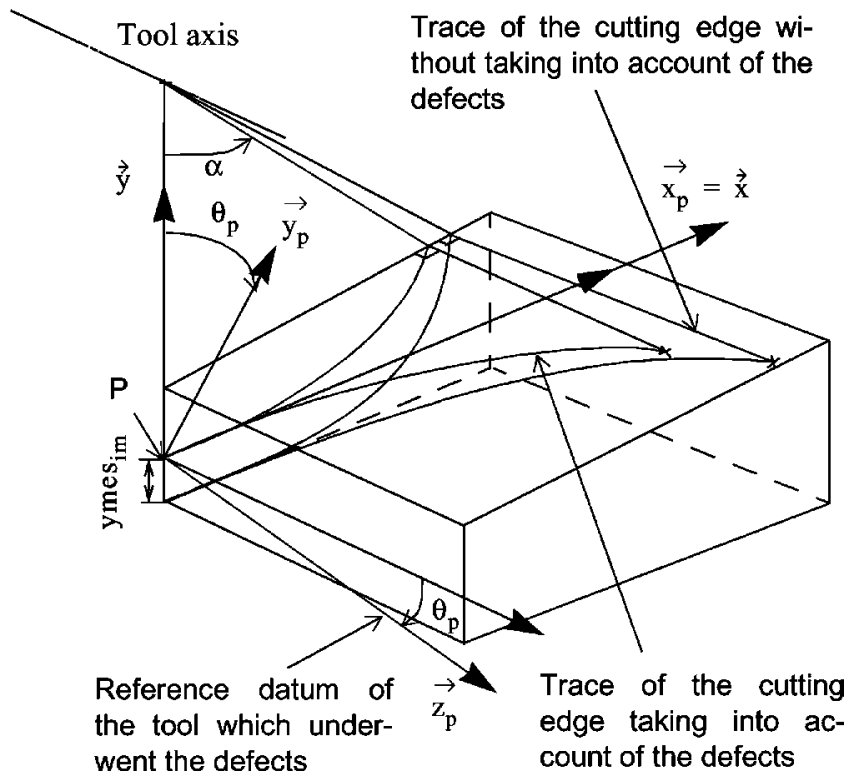

Fig. 12. Calculation of the maximal cutting edge engagement.
The entrance point of a cutting edge in the workpiece material is also calculated by intersection between the parametric curve previously defined and the sides of the workpiece faces. The determination of such a point allows to determine the minimal engagement angle.

Once the values measured after machining of the calibration plane are known, the calculation of the real engagement angles can easily be carried out within a $\mathrm{CAD} / \mathrm{CAM}$ environment by using the preceding developments.

\subsection{Numerical results}

The taking into account of the slope defect and of the deflection defect in the identification procedure is very significant. This result is visible on Fig. 13 which compares the error between the simulated points cloud and the measured points cloud taking the deviation and the slope of the tool on point $P$ in the calculation of $\alpha$ into account or not.

With the taking into account of the defection defect and of the slope defect, the differences are regularly distributed between -0.04 and $0.05 \mathrm{~mm}$ on all the surfaces. By neglecting these defects, some more significant variations of -0.06 up to $-0.08 \mathrm{~mm}$ appear in three zones of the machined surface.

\section{Application}

The tool workpiece couple was identified with the preceding procedure. The application consists of carrying out simple machining by calculating a prediction of the expected surface. Machining is carried out with a tool path theoretically generating the nominal form. The machined surface is measured and compared with the expected surface.

The application proposed consists of machining a circular interpolation on a milling center. Taking the curve into account, the engagement angle is smaller than in the case of a plane for a given radial engagement. The difficulty of predicting the defects relates to the fact that the tool deflection necessary for the calculation of the effective engagement angles cannot be estimated any more from the measured surface. We thus suppose that the tool deflection is continuous during the sweeping of the surface and we then calculate the deviation in a given section starting from the profile calculated for the preceding one. The engagement angles are thus calculated starting from this deviation and from the radial engagement of the section studied, always by intersection with the helix representing the cutting edge.

Fig. 14 shows that the error between the profile measured and the profile simulated is lower or equal to 0.05 $\mathrm{mm}$ in absolute value on $96 \%$ of the machined surface; this proves that the hypotheses of defects realized with 
Taking into account of the defects

Milling direction

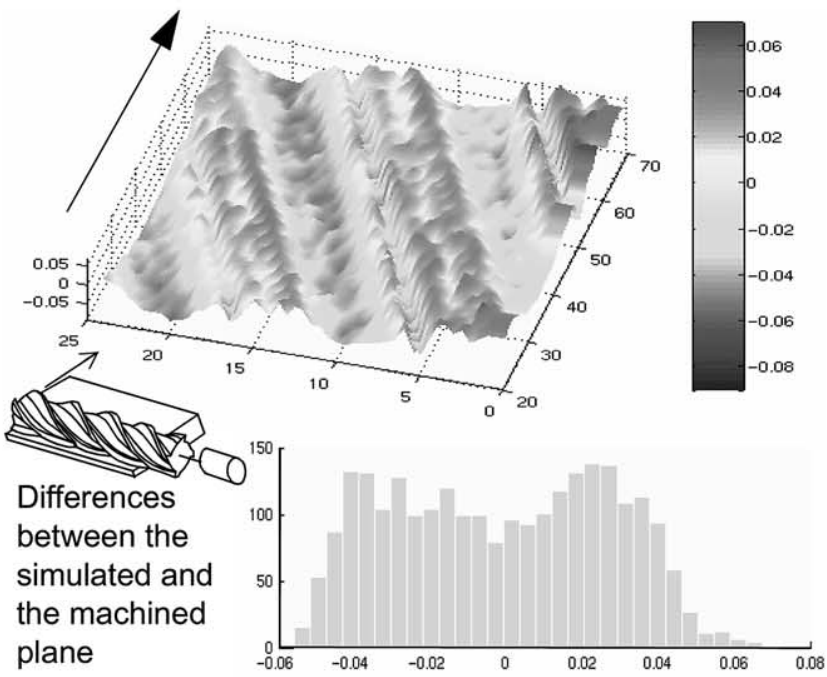

No taking into account of the defects

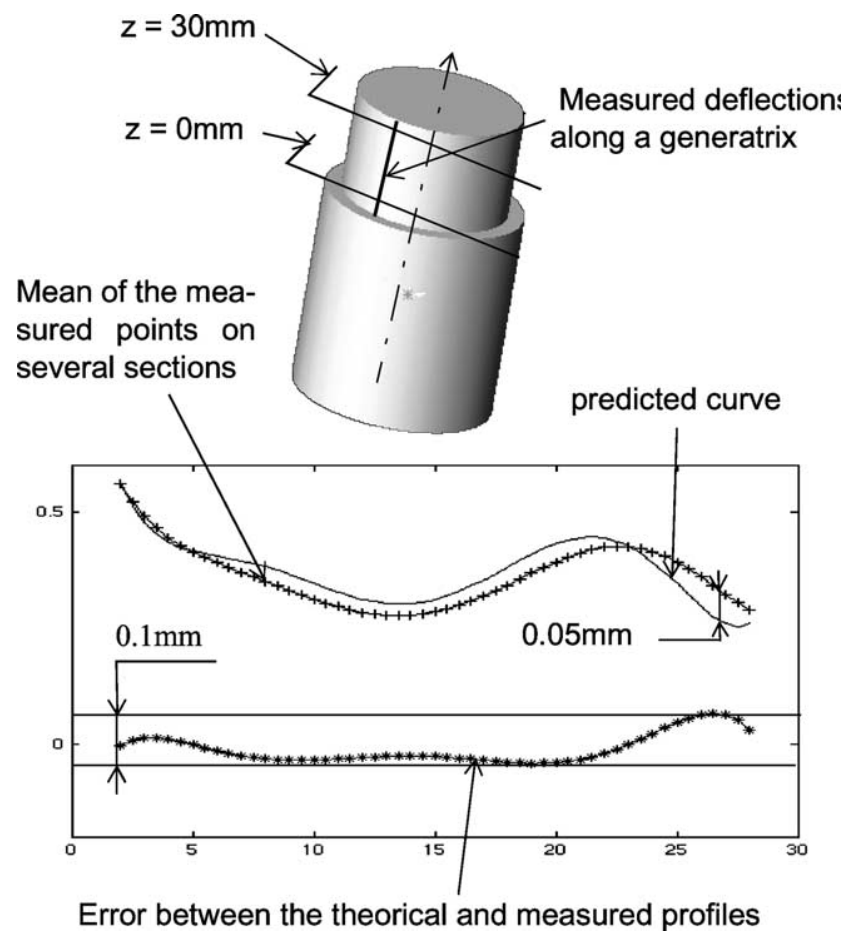

Fig. 14. Validation of the identification procedure.

\section{Milling direction}

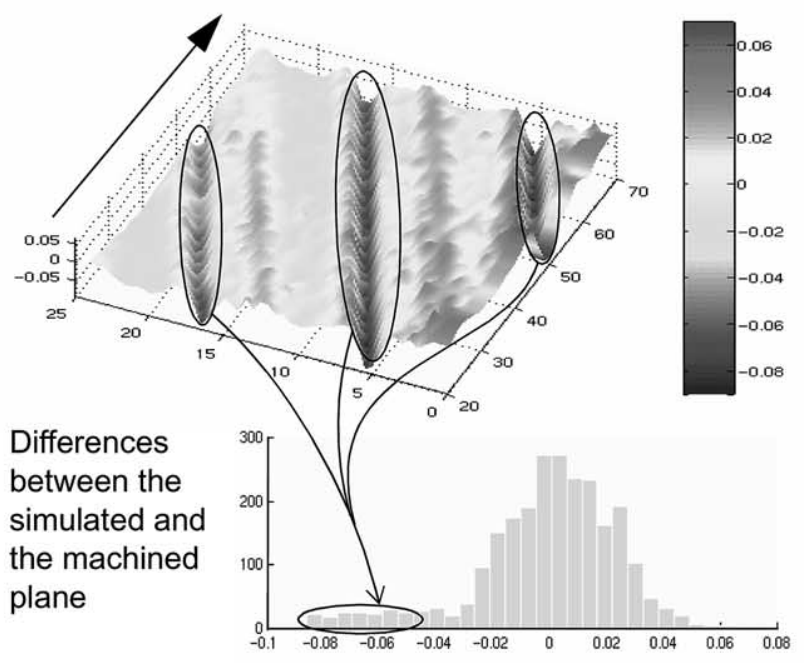

Fig. 13. Influence of the taking into account of the slope defect on the results of the identification.

the method proposed in the article is not impaired by a change in the tool engagement conditions. Taking the expected precision objectives into account, the static model previously described is validated since it makes it possible to predict the tool deflection defect, the 5\% remainder being ascribable to a phenomenon not yet identified.

\section{Conclusion-prospects}

The identification procedure of a tool workpiece couple proposed in this article is a procedure which takes the industrial constraints of time and cost into account.

Several applications were treated on simple surfaces, including with changes of the depth of cut. The results obtained were always satisfactory with the same order of magnitude of uncertainty on the hypothesis. The model proposed thus seems acceptable. It would now be necessary to develop a campaign of tests on a greater diversity of tool and parts materials with different tool geometries, in particular with odd numbers of teeth that give a quadratic moment Igy which is not constant.

Our current research tends to generalize the hypothesis step for complex surfaces and for curvature changes and radial engagement changes.

The test protocol proposed appears perfectly applicable in an industrial context on the production machine tool itself. We are currently developing the technique to establish the prediction model in a CAD system in order to allow the qualification of a tool and of its process according to the precision wanted for the whole surface for a raw part also defined by a CAD model. This result should then make it possible to implement machining tool paths corrections, in order to make up for the tool deflection so that the machined surface approaches the expected nominal form as much as possible. 


\section{References}

[1] E. Duc, Usinage des formes gauches, contribution à l'amélioration de la qualité des trajectoires d'usinage, Thèse de doctorat de l'Ecole Normale Supérieure de Cachan, France, 1998 [in French].

[2] H. Tönshoff, Optimal tool positionning for five-axis flank milling of arbitrary shaped surfaces, Production Engineering 7 (1) (2000).

[3] F. Monies, J.M. Redonnet, W. Rubio, P. Lagarrigue, Improved positionning of a conical mill for machining ruled surfaces, Journal of Engineering Manufacture 214 (7) (2000) 625-634.

[4] C. Lartigue, E, Duc, A. Affouard, Tool path deformation in 5axis flank milling using envelope surface, Computer Aided Design (2002), to appear.

[5] A. Firas, G. Coffignial, P. Lorong, M. Touratier, An explicit 2D thermo-mechanical finite element software for simulating chip formation and chip flow in machining, in: Third international conference on metal cutting and high speed machining Metz (France), 27-29 June, 2001.

[6] A. Moufki, A. Devillez, D. Dudzinski, A. Molinari, Thermomechanical modelling of cutting and experimental validation, in: Third international conference on metal cutting and high speed machining Metz (France), 27-29 June, 2001.

[7] Y. Altintas, Modeling approaches and software for predicting the performance of milling operations at MAL-UBC, Manufacturing Automation Laboratory (MAL), http://www.mech.ubc.ca/ mal.

[8] F. Abrari, Multi-Axis milling of flexible parts, PhD Thesis of McMaster University (Canada), 1998.

[9] H. Paris, G. Peigné, Modélisation de la génération de la surface usinée lors d'une opération de fraisage den UGV, in: Second Assises Machines et Usinage à Grande Vitesse Lille (France), 13-14 Mars, 2002, pp. 307-316 [in French].

[10] L. Hiaizhong, L. Xiaoping, Modelling and simulation of chatter in milling using a predictive force model, International Journal of Machine Tools Manufacture 40 (2000) 2047-2071.

[11] Y. Altintas, E. Budak, Analytical prediction of stability lobes in milling, Annals of the CIRP 44 (1) (1995) 357-362.

[12] J. Tlusty, Dynamics of high-speed milling, Journal of Engineering for Industry 108 (1986) 59-67.

[13] S. Smith, J. Tlusty, Efficient simulation programs for chatter in milling, Annals of the CIRP 42 (1) (1993) 463-466.

[14] L. Arnaud, G. Dessein, Application de la théorie des lobes de stabilité au fraisage de parois minces, in: Fourth international conference on integrated design and manufacturing in mechanical engineering, Clermont Ferrand (France), 14-16 May, 2002.

[15] E. Beauchesne, Simulation numérique de l'usinage à l'échelle
Macroscopique: prise en compte d'une pièce déformable, Thèse de doctorat de l'Ensam Paris, France, 1999 [in French].

[16] W.A. Kline, R.E. DeVor, J.R. Lindbergn, The prediction of cutting forces in end milling with application to cornerong cuts, International Journal of Machining Tool Design Research 22 (1) (1982) 7-12.

[17] T.-I. Seo, Intégration des effets de déformation d'outil en génération de trajectoires d'usinage, Thèse de doctorat de l'Ecole Centrale de Nantes, France, 1998.

[18] S. Jayaram, S.G. Kapoor, R.E. DeVor, Estimation of the specific cutting pressures for mechanistic cutting force models, International Journal of Machine Tools Manufacture 41 (2001) 265-281.

[19] J.J. Junz Wang, C.M. Zheng, Identification of shearing and ploughing cutting constants from average forces in ball-end milling, International Journal of Machine Tools Manufacture 42 (2002) 695-705.

[20] J.J. Junz Wang, C.M. Zheng, An analytical force model with shearing and ploughing mechanisms for end milling, International Journal of Machine Tools Manufacture 42 (2002) 761-771.

[21] B.W. Ikua, H. Tanaka, F. Obata, S. Sakamoto, K. Takeyasu, I. Tatsuo, Prediction of cutting forces and machining error in ball end milling of curved surfaces-2 Experimental verification, precision engineering, Journal of the International Societies for Precision Engineering and Nanotechnology 26 (2002) 69-82.

[22] E. Budak, Y. Altintas, E.J.A. Armarego, Prediction of milling force coefficients from orthogonal cutting data, Transactions of the ASME 118 (1996) 216-224.

[23] K. Cheng, X.W. Liu, D. Webb, X.C. Luo, Prediction of cutting force distribution and its influence on dimensional accuracy in peripheral milling, International Journal of Machine Tools Manufacturet 42 (2002) 791-800.

[24] W.S. Yun, D.W. Cho, Accurate 3D cutting force prediction using cutting-condition-independent coefficients in end milling, International Journal of Machine Tools Manufacture 41 (4) (2001) 463-478.

[25] Y. Landon, G. Cohen, J.M. Redonnet, P. Lagarrigue, Elaboration d'une pièce master pour la caractérisation du défaut de positionnement de l'outil en travail, in: Fourth international conference on integrated design and manufacturing in mechanical engineering Clermont Ferrand (France), 14-16 May, 2002.

[26] A. Larue, B. Anselmetti, Contribution to the tool paths generation in cam system, in: Fourth international conference on integrated design and manufacturing in mechanical engineering Clermont Ferrand (France), 14-16 May, 2002.

[27] J.G. Choi, M.Y. Yang, In-process prediction of cutting depths in end milling, International Journal of Machine Tools Manufacture 39 (1999) 705-721. 